\title{
Determinación de trazas de Molibdeno (VI), Níquel (II) y Vanadio (V) en cementos por polarografía diferencial de impulsos
}

\author{
ANTONIO COBO-GUZMAN (*), RAFAEL GALLEGO-ANDREU $(*)(+)$ y DEMETRIO GASPAR-TEBAR (**)
}

\author{
$R E S U M E N$ \\ En el presente trabajo se da cuenta de los resultados \\ obtenidos cuando se aplica la polarografia diferencial \\ de impulsos a la determinación de los contenidos \\ de compuestos a base de molibdeno (VI), níquel (II) \\ $y$ vanadio (V) en un cemento portland industrial; \\ dichos contenidos se encuentran en cantidades \\ del orden de p.p.m. \\ En los estudios realizados se ha puesto de manifiesto \\ que no es posible determinar simultáneamente el \\ contenido de los tres iones mencionados en el cemento, \\ pero que trabajando en medio ácido (clorhidrico o \\ sulfúrico), en medio amoniacal o cianurado y en medio \\ básico (producido por $\mathrm{NaHCO}_{3}$ ) se obtienen \\ polarogramas que permiten realizar las mencionadas \\ determinaciones cuantitativas de Mo (VI) en el primer \\ medio, de Ni (II) en el segundo y de V(V) en el tercero. \\ Gracias a la alta sensibilidad de la polarografia ha \\ sido posible determinar la concentración de Ni (II) \\ en el cemento estudiado, que se encuentra en cantidades \\ comprendidas entre 2,9 y 3,4 p.p.m. $\left(0,0002_{9} y\right.$ \\ $0,0003_{4} \%$, en masa).
}

\section{$S U M M A R Y$}

In this work an account is given of the results obtained when the differential pulse polarography' (differential pulse anodic stripping voltametry) is applied to the determination of the contents of the compounds by molybdenum (VI), nickel (II) and vanadium (V) in a common portland cement which are in quantities of the order of p.p.m.

Those studies show that it is not possible to determine simultaneaously the content of the three mentioned ions in the cement, but if working in acid medium (hydrochloridric and sulphuric), in ammoniacal or cyanided medium and in basic medium (produced by $\mathrm{NaHCO}_{3}$ ) polagrogrammes are obtained which allow to complete those mentioned determinations of $M o(V I)$ in the first medium, of $\mathrm{Ni}(\mathrm{II})$ in the second and of $V(V)$ in the third. Because of the high sensibility of the polarography it is possible to determine the $\mathrm{Ni}(\mathrm{II})$ which is found in quantities from 2.9 to 3.4 p.p.m. (0.0002, to $0.0003_{4} \%$, in mass).

\section{INTRODUCCION}

La polarografia diferencial de impulsos permite la determinación de trazas de determinados iones en concentración final $10^{-7} \mathrm{M}$, lo que supone para la relación sólido/sólido, cantidades de p.p.m. En el presente artículo se aplica para determinar trazas de niquel (II), vanadio (V) y molibdeno (VI) en un cemento portland industrial.

Esta técnica se ha usado anteriormente para determinar trazas de plomo, cobre, cadmio y cinc en CMC y bismuto en cobre (1).

Deane (2) describe un procedimiento de electrolisis diferencial (polarografia diferencial de impulsos) para determinar los compuestos de talio en el polvo de los hornos de las fábricas de

(*) Departamento de Quimica Analitica del CSIC; Facultad de Ciencias Quimicas de la Universidad Complutense de Madrid.

(**) Instituto Eduardo Torroja de la Construción y del Cemento (CSIC).

(+) Fallecido. 
cemento y en los óxidos de hierro. Los resultados obtenidos por medio de esta técnica y por espectrometria de llama son análogos, aun cuando por la primera se pueden dosificar cantidades menores de 0,1 p.p.m.

\section{REVISION BIBLIOGRAFICA}

Stackelberg y otros (3) hallaron que el molibdeno (VI) no da onda polarográfica en medio neutro o alcalino, y que en medio sulfúrico se obtiene una onda. Uhl (4) trabajó en un medio compuesto por los ácidos nítrico, oxálico y láctico, obteniendo dos ondas. Nichols y Rogers (5) obtuvieron ondas de molibdeno (VI) en medio nitrico-nitrato amónico, encontrando que tanto el $\mathrm{pH}$, asi como la concentración de ion nitrato, influyen sobre la altura de onda. Johnson y Robinson (6) encontraron tres ondas en medio sulfúrico-sulfato de sodio, correspondientes al paso de Mo (VI) a Mo (V) la primera y las otras dos a Mo (III); para concentraciones relativamente bajas de molibdeno (VI) se mide la concentración por la altura de la primera onda. Kanevskii y otros (7) estudiaron el comportamiento del molibdeno (VI) que da dos ondas cuando la concentración de fosfórico es $1 \mathrm{M}$ y tres ondas cuando el ácido es $10 \mathrm{M}$. Protsenko y Kovalenko (8) determinaron polarográficamente molibdeno (VI) en materiales de níquel, utilizando para tal fin el medio nitrico, en el que no encontraron interferencias de níquel (II) y vanadio (V). Partani y Miglorini (9) determinaron molibdeno en medio pirofosfórico; en este medio el Mo (VI) se reduce a Mo (V) y Mo (III). Kolthoff y Hodara (10) estudiaron la reducción de Mo (VI) en medio sulfúrico, obteniendo dos ondas; si la concentración de dicho ácido es superior a 2,5 M la primera onda se desdobla en dos, que los autores atribuyeron a la reducción de dos especies diferentes de Mo (VI) a Mo (V). Si la concentración de ácido es inferior a $0,1 \mathrm{M}$ es la segunda onda la que se desdobla; atribuyéndolo a la reducción de formas polimeras de Mo (V). En otro trabajo, los mismos autores (11) encuentran que la presencia de wolframio aumenta la altura de la onda catalítica de molibdeno (VI) en presencia de perclorato, nitrato o clorato, siendo este efecto más acusado en el caso del perclorato. Kawahara y otros (12) dieron los resultados para molibdeno (VI), wolframio (VI) y titanio (IV) en medio fosfórico desde 1 a $12 \mathrm{M}$. El molibdeno (VI) da tres ondas; el wolframio (VI) da una onda próxima a la segunda del molibdeno (VI) y el titanio (IV) da una onda próxima a la primera del molibdeno (VI). Höltje y Geyer (13) estudiaron los polarogramas de molibdeno (VI) en medios fosfórico, clorhídrico y sulfúrico, obteniendo de dos a cuatro ondas, atribuídas a los pasos $\mathrm{Mo}(\mathrm{VI}) \rightarrow \mathrm{Mo}(\mathrm{V})$; Mo $(\mathrm{V}) \rightarrow$ Mo $(\mathrm{III})$ rojo; Mo $(\mathrm{V}) \rightarrow$ Mo $(\mathrm{III})$ verde y Mo $(\mathrm{VI}) \rightarrow \mathrm{Mo}(\mathrm{OH})_{3}$.sólido y que las disoluciones alcalinas de molibdato no son reducibles en el electrodo de gotas. Desmukh y Srivastava (14) determinaron uranio (VI) y molibdeno (VI) en medio clorhídrico, reduciendo previamente con clorhidrato de hidrazina, con lo que ambos elementos pasan a valencia cinco. En el polarograma se obtienen dos ondas, la primera corresponde al uranio y la segunda al molibdeno; ambos pasan de valencia cinco a cuatro. Athawale y otros (15) determinaron molibdeno en aleaciones uranio-molibdeno en fondo cloruro de potasio, en el que el vanadio interfirió exaltando la onda del molibdeno, lo cual se evitó añadiendo citrato al fondo. Afghan y Dagnall (16) determinaron molibdeno previa extracción con éter del complejo molibdeno (VI) sulfocianuro, registrando el polarograma usando la fase orgánica; en este medio etéreo sólo el molibdeno da onda. Polotebnona y Furtune (17) estudiaron la polarografía de los ácidos molibdovanadofosfórico y molibdovanadosilícico en ácido sulfúrico, obteniendo dos ondas de reducción, que corresponden la primera a la reducción del vanadio y la segunda a la del complejo trinuclear P-Mo-V o Si-Mo-V. Bikbulatova y Synyakova (18) estudiaron el comportamiento del molibdeno por polarografia en medio clorhídrico $0,01-0,1 \mathrm{~N}$, encontrando tres ondas para concentraciones de molibdeno (VI) comprendidas entre $5 \times 10^{-5}$ y $1 \times 10^{-4} \mathrm{M}$; para concentración mayor de molibdeno (VI), la primera onda desaparece, en la segunda aparece un máximo muy acusado, que no se puede suprimir con gelatina y la tercera onda coincide con la descarga del hidrógeno. Ogura y Enaka (19) estudiaron el mecanismo de la reducción polarográfica de molibdeno (VI) en presencia de citrato, observando que el complejo molibdeno (VI) citrato se reduce a $\mathrm{MoO}^{3+}$. Sharipova y otros (20) estudiaron la reducción polarográfica del 
peróxido de hidrógeno catalizada por molibdeno (VI), hallando que a $\mathrm{pH} 1$ predomina el polimero hexámero. El grado de polimerización del molibdeno (VI) disminuye con el $\mathrm{pH}$; cuando el $\mathrm{pH}$ es $2,5-4,5$ predomina la forma dimera y a $\mathrm{pH} 5$ el molibdeno (VI) no está polimerizado. Stach y Schoene (21) determinaron trazas de molibdeno (VI) en presencia de iones perclorato o nitrato en medio sulfúrico, midiendo la onda catalitica obtenida en el polarograma; para concentración $10^{-7} \mathrm{M}$ en molibdeno (VI), el error fue del $10 \%$ y para $10^{-5} \mathrm{M}$ del 3-5\%. Ogura $\mathrm{y}$ otros (22), anteriormente citados, determinaron molibdeno (VI) en presencia de ácido glicólico, láctico o málico. Lugoroi y Paklerova (23) estudiaron el mecanismo de la reducción polarográfica de los heteropoliácidos silicomolibdowolfrámicos, en distintas proporciones de silicio, molibdeno y wolframio, observando tres ondas; siendo la tercera la que corresponde a la reducción de Mo (IV). Pabbu Vijay y otros (24) determinaron molibdeno (VI) en agua de mar, separando previamente el molibdeno (VI) mediante coprecipitación con sulfuro de cadmio, operando finalmente en medio perclorato-EDTA $(\mathrm{pH}=6)$, obteniendo una onda; la precisión para $5 \mu \mathrm{g} / \mathrm{ml}$ es del $13 \%$. Efrain y otros (25) investigaron la polarografia de molibdeno (VI), uranio (VI), hierro (III) y arsénico (V) en presencia de vanadio (V) utilizando de fondo ácido sulfúrico en presencia de resorcina o pirogalol. Edmonds (26) determinó molibdeno (VI) por polarografia diferencial de impulsos en ácido nítrico 0,06 $\mathrm{M}$ como electrolito soporte; entre otras interferencias cita al titánio (IV) en concentración superior a $0,5 \mathrm{mg} / \mathrm{l}$. Lanza y otros (27) determinaron molibdeno en aceros utilizando como electrolito soporte nítrico-nitrato de amonio; la precisión fue del $5 \%$.

Las investigaciones de los polarogramas de vanadio en sus diversos estados de valencia fueron llevados a cabo por Zelter y otros (28), Stackelberg y col. (29), Thanheisser y Willens (30), Voriskova y col. (31), Lingane (32) y Lignane y Meites (33). Estos últimos estudiaron la reducción polarográfica de vanadio (V) en medio clorhidrico y sulfúrico. En ambos casos, el vanadio se reduce en dos etapas; pasando en la primera de valencia 5 a 4 y en la segunda de 4 a 2 . La primera onda se desarrolla mal, porque el vanadio (V) oxida al mercurio. La segunda onda se desarrolla mejor en medio sulfúrico que clorhídrico. Si la concentración del ácido es relativamente muy alta, la descarga del ion hidrógeno interfiere a la segunda onda.

En medio amoniacal, el vanadio (V) da dos ondas muy próximas. En medio oxalato $(\mathrm{pH}=4,6)$ el vanadio $(\mathrm{V})$ da dos ondas; la primera también está interferida por la oxidación del mercurio. Thanheiser y Willens (30) vieron que el vanadio (V) en medio fuertemente alcalino, fondo hidróxido de litio, solamente da una onda mal definida.

El vanadio en valencia 4 da, en medio sulfúrico, una onda correspondiente a la reducción a valencia 2. Esta onda está mal definida e interferida por la descarga de ion hidrógeno. En medio ácido más diluído, la onda se desplaza a potenciales más negativos y a $\mathrm{pH} 6$ se obtiene una doble onda mal definida. En medio fuertemente alcalino - hidróxido de sodio- no da onda de reducción, pero si da una onda de oxidación.

Polotebnona y Furtune (17), ya citados en el apartado correspondiente al molibdeno, estudiaron el ácido molibdovanadofosfórico y molibdovanadosilícico, con los resultados ya consignados. Lucena y otros (34) estudiaron la determinación de vanadio en aceros, reduciendo previamente al vanadio (V) con sulfito a vanadio (IV) y utilizando cianuro como electrolito soporte. El níquel en concentraciones inferiores a diez veces la de vanadio no interfirió; el molibdeno sí. Grigor'eva y otros (35) determinaron vanadio (IV y III) en presencia de sulfocianuro en el intervalo de $\mathrm{pH}$ 1-5; el vanadio en valencia 4 da dos ondas y en valencia 3 una onda. El anión fosfato en concentración mil veces superior al vanadio no interfirió. Nomura y Nakagawa (36) determinaron vanadio $(\mathrm{V})$ por oxidación catalitica del ortoaminofenol, operando en medio cloratoperclorato a $\mathrm{pH} 2$; el molibdeno interfiere. Rao y otros (37) determinaron vanadio (1-6 $\mu \mathrm{M})$ en medio acético-acetato de $\mathrm{pH} 5$ por la reducción polarográfica catalitica del bromato.

El níquel (II) se puede determinar en fondo nitrato, perclorato o medio ligeramente ácido dando 
una onda bien definida. Pavlick y col. (38) obsevaron que el niquel (II) en presencia de cloruro se reduce a potencial más positivo que el ion hexamoniquel. Lingane y Kerlinger (39) vieron que los complejos de níquel (II) con sulfocianuro y piridina se reducen a un potencial más positivo que el ion hexacuoniquel; estos medios son recomendables para determinar níquel en presencia de cobalto, el cual da su onda unos 0,3 voltios más negativa. El sulfocianuro también se recomienda por Stout, Levy y col. (40) para determinar níquel en presencia de cantidades grandes de cinc. En medio amoniacal el níquel (II) da una onda bien desarrollada y este medio se puede utilizar para determinar niquel (II) en presencia de cobalto (II) y cinc (II). Hume y Kolthoff (41) estudiaron el comportamiento del complejo níquel-cianuro en fondo cloruro o hidróxido de sodio, obteniendo una onda bien definida; el fondo cianuro es útil para el níquel (II) en presencia de cobre (II) y cinc (II), los cuales no dan onda en ese medio. El níquel (II) no da onda en presencia de tartárico.

En el Departamento de Química Analítica del C.S.I.C. (42) se estudió la determinación polarográfica de cobre (II), níquel (II), cinc (II) y cobalto (II). Para determinar níquel (43) entre otros, como impureza del plomo, se utilizó como disolución de fondo sulfato, carbonato e hidróxido de amonio. En fondo sulfato de litio (42) el níquel (II) se puede determinar en presencia de cobalto (II), pero el cinc (II) interfiere.

Ruvinskii y otros (44) estudiaron las preondas catalíticas del níquel (II) en presencia de productos intermedios de la sintesis de la vitamina $\mathrm{B}_{6}$, hallando que estos productos aceleran la descarga del niquel (II), si bien ésto lo aplican a la determinación de esos productos.

Astafeva y otros (45) estudiaron el comportamiento de los complejos de cobre (II), níquel (II) y cobalto (II) con la dimetilglioxima, dando las condiciones óptimas para la determinación de dichos iones a partir de la onda catalítica del hidrógeno; las condiciones para el níquel fueron cloruro de amonio $1 \mathrm{M}$, pH 8 y dimetilglioxima $1,3 \times 10^{-4} \mathrm{M}$. Kuhnhardt y Agermamm (46) determinaron plomo, cadmio, niquel y cinc en cobre, previa separación por medio del cambiador de iones Wofatit; el cobre (II) se enmascaró con tiosulfato y los iones metálicos fueron eluídos con ácido nitrico $1 \mathrm{M}$, utilizando cloruro de potasio $0,5 \mathrm{M}$ - piridina $0,5 \mathrm{M}$ como fondo para níquel (II) y cinc (II) y bromuro de potasio $0,5 \mathrm{M}$ - ácido bromhídrico $0,1 \mathrm{M}$ para plomo (II), cadmio (II) y cinc (II). El níquel (II) también se determinó como complejo con dipiridilo. Mambetkaziew (47) estudió la naturaleza de las preondas de los polarogramas de níquel (II) en presencia de 2-2' bipiridina en fondo nitrato de potasio $1 \mathrm{M}$. Ruvinskii y otros (48) estudiaron los efectos catalítico e inhibidor de las vitaminas del grupo $\mathrm{B}$ sobre las ondas polarográficas de níquel (II) en acetato de sodio $0,1 \mathrm{M}$ y pH 6,5-7. Las vitaminas $B_{3}, B_{12}$, ácido fólico y nicotinamida catalizan la electrorreducción del niquel (II); las vitaminas $\mathrm{B}_{1}$ y $\mathrm{B}_{2}$ inhiben la reacción y la $\mathrm{B}_{6}$ no tiene efecto. Los mismos Ruvinskii y Sirko (49) estudiaron el papel de los agentes complejantes durante la reducción electrocalítica de níquel (II) en presencia de cisteína y glutationa, en fondo cloruro de potasio y tampón bórico-borato $(\mathrm{pH} \sim 7)$. La cisteina tiene una actividad algo mayor que la glutationa. Voitenko y otros (50) estudiaron la determinación polarográfica de niquel (II) en cloruro de cobalto. El contenido en niquel (II) fue por debajo de 0,05 p.p.m. El producto secado $\mathrm{y}$ disuelto en metanol y ácido clorhídrico $\mathrm{M}$ se pasa por columna AV 17-X8 para retener el cobalto (II); el níquel (II) se eluye con ácido clorhídrico. El electrolito soporte fue tampón amoniacal $0,1 \mathrm{M}$ de $\mathrm{pH} \mathrm{9,25}$ y dimetilglioxima $0,1 \mathrm{M}$. La relación entre la altura de onda y la concentración de níquel (entre 1 a $100 \mathrm{ng} \mathrm{m}^{-1}$ ) fue lineal. El error fue 13,4 \% para 0,4-135 p.p.m. de niquel en la muestra.

\section{PARTE EXPERIMENTAL}

\subsection{Instrumental}

a) Polarógrafo Polarecord E 506. Metrohm Herisau. 
b) Electrodo de trabajo: electrodo de mercurio.

El mercurio, para tal efecto, se utiliza bi o tridestilado. El problema que puede plantear el mercurio es el de las trazas de cobre, plomo, cinc y cadmio, que casi siempre le acompañan como impurezas. Estas impurezas, debido a su concentración, pueden no tener importancia en la polarografía clásica o diferencial de impulsos, pero sí la tienen cuando se emplea la técnica de redisolución anódica, debido a la mayor sensibilidad de esta última.

c) Electrodo de referencia: electrodo de plata-cloruro de plata, con cloruro de potasio a saturación.

d) Electrodo auxiliar: electrodo de platino.

\subsection{Reactivos}

a) Acido clorhídrico, $\mathrm{HCl}$; Merck, p.a.

b) Acido sulfúrico, $\mathrm{H}_{2} \mathrm{SO}_{4}$; Merck, p.a.

c) Carbonato de amonio, $\left(\mathrm{NH}_{4}\right)_{2} \mathrm{CO}_{3}$; Merck, p.a.

d) Hidróxido de amonio, $\mathrm{NH}_{3} \cdot \mathrm{H}_{2} \mathrm{O}$; Merck, p.a.

e) Molibdato de amonio tetrahidratado, $\left(\mathrm{NH}_{4}\right)_{6} \cdot \mathrm{Mo}_{7} \mathrm{O}_{24} \cdot 4 \mathrm{H}_{2} \mathrm{O}$; Merck, p.a.

f) Sulfato de niquel heptahidratado, $\mathrm{NiSO}_{4} .7 \mathrm{H}_{2} \mathrm{O}$; Merck, p.a.

g) Cianuro de potasio, KCN; Merck, p.a.

h) Carbonato de sodio anhidro, $\mathrm{Na}_{2} \mathrm{CO}_{3}$; Merck, p.a.

i) Carbonato ácido de sodio, $\mathrm{NaHCO}_{3}$; Merck, p.a.

j) Hidróxido de sodio, $\mathrm{NaOH}$; Merck, p.a.

k) Oxido de vanadio (V), $\mathrm{V}_{2} \mathrm{O}_{5}$; Merck, p.a.

\subsection{Disoluciones patrones}

Se han utilizado disoluciones patrones $10^{-2}$ y $10^{-3} \mathrm{M}$ de molibdeno (VI), de niquel (II) y de vanadio $(\mathrm{V})$, la primera de las cuales $\left(10^{-2} \mathrm{M}\right)$ se ha preparado disolviendo:

a) $12,3595 \mathrm{~g} / l$ de molibdato de amonio tetrahidratado en agua permutada y bidestilada,

b) $2,8086 \mathrm{~g} / \mathrm{l}$ de sulfato de níquel heptahidratado en agua permutada y bidestilada,

c) $1,8190 \mathrm{~g} / \mathrm{l}$ de óxido de vanadio $(\mathrm{V})$ en agua permutada y bidestilada.

La segunda $\left(10^{-3} \mathrm{M}\right)$ por disolución a partir de la primera, de tal modo que $1 \mu l$ de la disolución $10^{-3} \mathrm{M}$ se corresponde con $10^{-9}$ moles y $50 \mu l$ a $5 \times 10^{-8}$ moles.

\subsection{Disolución de la muestra de cemento}

Se pesan de 0,5 a $5 \mathrm{~g}$, respectivamente, del cemento estudiado y se tratan con $25 \mathrm{~m} l$ de clorhidrico $(1: 1)$ o sulfúrico $(1: 1)$; a continuación, se filtra y se lava con agua permutada y bidestilada caliente el residuo hasta que el líquido de lavado no dé reacción de cloruros o sulfatos, respectivamente; la fase líquida, junto con las aguas de lavado, se llevan a un matraz aforado de 100 ó $250 \mathrm{~m} l$ según la cantidad de muestra atacada y, por último, se enrasa el matraz con agua bidestilada. 
Debido a los contenidos de los iones a estudiar [V (V), Mo (VI) y Ni (II)] y la sensibilidad del aparato, los mejores resultados se obtuvieron a partir de $5 \mathrm{~g}$ de muestra y aforados a $100 \mathrm{~m} l$.

\subsection{Estudios previos}

Con objeto de obtener unas condiciones de trabajo apropiadas que permitiesen determinar cuali y cuantitativamente los compuestos de molibdeno (VI), vanadio (V) y níquel (II) presentes en el cemento, por polarografía diferencial de impulsos, se ha realizado una serie de trabajos, cuyos resultados se discuten a continuación, utilizando el procedimiento operatorio que se incluye en el apartado 3.6. Por otra parte, se han estudiado diferentes disoluciones de fondo, las cuales proporcionan a su vez distintos $\mathrm{pH}$, con objeto de modificar el potencial de semionda caracteristico de los mencionados iones y proceder asi a determinar sus concentraciones.

Los resultados obtenidos con los medios o disoluciones de fondo seleccionados experimentalmente han sido los siguientes:

\subsubsection{Disolucion clorhídrica o sulfúrica $(p H<7)$}

a) El polarograma correspondiente a la muestra de cemento tratada con $\mathrm{HCl}(1: 1)$ o con $\mathrm{H}_{2} \mathrm{SO}_{4}(1: 1)$, como se señala en 3.4., presenta inicialmente una perturbación que puede interpretarse como la existencia de dos picos muy próximos, debido al ataque del electrodo, por el cual el mecanismo se oxida a mercurio (I) ó (II); esta perturbación es tanto más acusada cuanto mayor es la concentración de ácido. Por otra parte, en esta zona de potenciales el vanadio $(\mathrm{V})$ cataliza el ataque y al final se obtiene la barrera del salto del hidrógeno, que limita o imposibilita ver picos próximos a dicha barrera; así mismo, en esta zona de potenciales, el níquel (II) cataliza el salto del hidrógeno, rebajando la sobretensión y limitando la zona de potenciales útiles de trabajo.

b) El molibdeno (VI) en los medios ácidos señalados puede dar tres picos en el polarograma, de los cuales, el primero aparece muy próximo al salto inicial y el tercero al final, siendo difíciles de medir; cosa que no sucede con el segundo.

c) El níquel (II) y el vanadio (V) dan, cada uno, un pico en el polarograma obtenido en estas condiciones, que, además de estar muy próximos al tercero de molibdeno (VI), casi coinciden entre sí.

\subsubsection{Disolución en medio alcalino}

En medio alcalino, amoniacal o cianurado, el molibdeno (VI) no da picos en el polarograma; si dicho medio alcalino se produce por la adición de hidróxido de sodio o carbonato de sodio a la disolución 3.4. los picos que aparecen del níquel (II) y del vanadio (V) no son útiles para esta finalidad, por lo que se desecharon estos reactivos.

\subsubsection{Disolución en medio amoniacal o cianurado}

En medio amoniacal [ $\mathrm{NH}_{4}$ (I) - $\left.\mathrm{NH}_{4} \mathrm{OH}-\mathrm{NH}_{3} \cdot \mathrm{H}_{2} \mathrm{O}\right]$ el níquel (II) da un pico bien definido y el vanadio $(\mathrm{V})$ apenas si interfiere; la presencia de yoduros en el medio produce una interferencia en el comportamiento polarográfico de ambos iones.

En medio cianurado $[\mathrm{CN}$ (I) $+\mathrm{OH}$ (I)] el níquel (II) da, como en medio amoniacal, un pico bien definido, mientras que el vanadio $(\mathrm{V})$ no da pico alguno. 


\subsubsection{Disolución en medio básico, producido por carbonato ácido de sodio}

Cuando el medio ácido de la disolución 3.4. se neutraliza con exceso de carbonato ácido de sodio hasta conseguir un $\mathrm{pH} \sim 8$, el vanadio $(\mathrm{V})$ da un pico bien definido, mientras que el molibdeno (VI) y el níquel (II) no dan picos.

\subsubsection{Conclusiones}

A partir de los polarogramas obtenidos se ha puesto de manifiesto que no es posible determinar simultáneamente el contenido de Mo (VI), Ni (II) y V (V) en el cemento, pero que las disoluciones de la muestra en medio ácido clorhídrico o sulfúrico, en medio amoniacal o cianurado y en medio básico (producido por $\mathrm{NaHCO}_{3}$ ) proporcionan polarogramas que permiten realizar la mencionada determinación cuantitativa de los compuestos a base de Mo (VI), Ni (II) y $V(V)$, respectivamente.

De aqui que se haya procedido como se detalla a continuación, apartado 3.6., para realizar dichas determinaciones.

\subsection{Determinación del contenido de compuesto de Mo (VI), Ni (II) y V (V) en el cemento}

\subsubsection{Técnica operatoria}

Teniendo en cuenta los resultados obtenidos en el apartado 3.5. se ha utilizado la disolución obtenida en 3.4. para determinar el contenido de compuestos de Mo (VI); para las determinaciones correspondientes a los compuestos de $\mathrm{Ni}$ (II) y $\mathrm{V}$ (V) se requiere un tratamiento posterior de la mencionada disolución obtenida según 3.4., que se describe en los apartados 3.6.3. y 3.6.4., respectivamente.

\subsubsection{Determinación del contenido de Mo (VI)}

Como se ha indicado en 3.6.1., la disolución obtenida en el apartado 3.4. no necesita tratamiento posterior para determinar el contenido de Mo (VI); para ello, se procede como sigue:

Se toma $1 \mathrm{~m} l$ de la disolución (lo mismo sulfúrica que clorhídrica) y $25 \mathrm{~m} l$ de agua permutada y bidestilada que se vierten directamente en la célula polarográfica, registrando a continuación el polarograma correspondiente (fig. 1, gráfica A, y fig. 2, gráfica A; medio sulfúrico y clorhidrico, respectivamente).

Para la determinación cuantitativa del Mo (VI), se procede por el método de adición standard; para ello una vez registrado el polarograma anterior, se adicionan a la disolución de la célula polarográfica $50 \mu \mathrm{l}$ de la disolución patrón de $\mathrm{Mo}(\mathrm{VI}) 10^{-3} \mathrm{M}$ (equivalente a $5 \times 10^{-8}$ moles) y se vuelve a registrar el polarograma (gráficas B de las figuras 1 y 2). El proceso de adición se repite cuantas veces se estime oportuno: por regla general, suele ser suficiente con 4 ó 5 adiciones (gráficas $\mathrm{C}$ y siguientes de las figuras 1 y 2 ).

En la figura 1 se han representado, a título de ejemplo, los polarogramas mencionados anteriormente trabajando con corriente continua (polarogramas de la zona derecha) y con corriente alterna (polarogramas de la zona izquierda). 


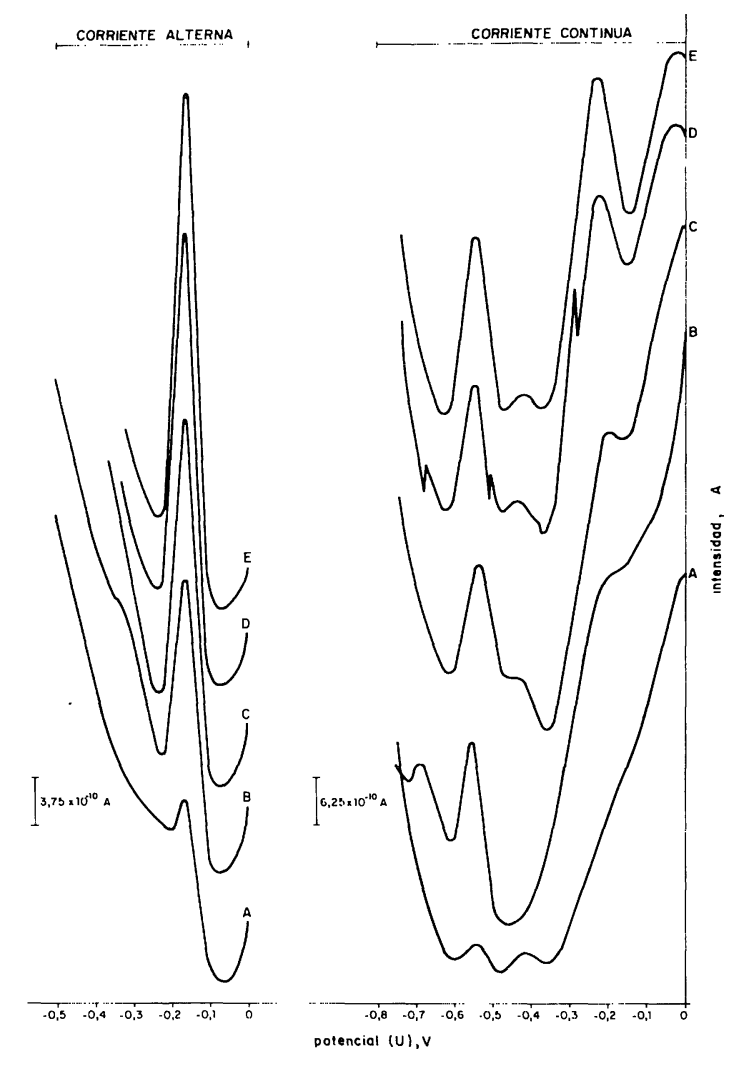

Fig. 1.-Determinación de Mo (VI). Polarogramas de: A.-Disolución del cemento, medio sulfúrico.

B, C, D y E.-Disolución del cemento, medio sulfúrico, más $n 50 \mu l$ de disolución de Mo (VI), $10^{-3}$ M. $n=1$, para $\mathrm{B}$, a 4 , para $\mathrm{E}$.

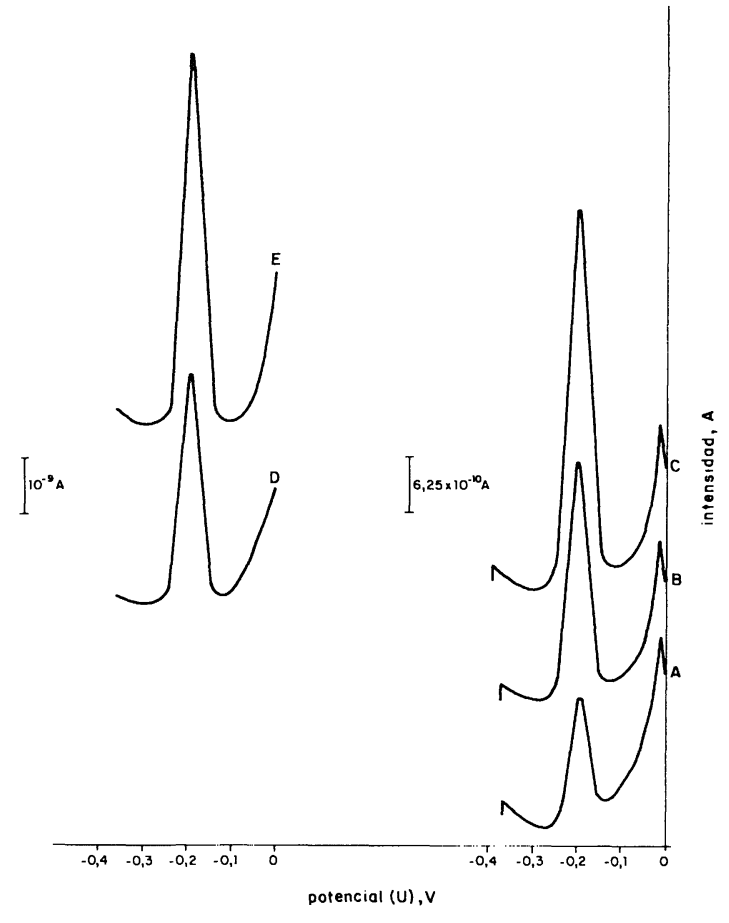

Fig. 2.-Determinación de Mo (VI). Polarogramas de: A.-Disolución del cemento, medio clorhídrico.

B, C, D y E.-Disolución del cemento, medio clorhidrico, más $n 50 \mu l$ de disolución de Mo (VI), $10^{-3} \mathrm{M}$. $n=1$, para $\mathrm{B}$, a 4 , para $\mathrm{E}$.

\subsubsection{Determinación del contenido de Ni (II)}

Para determinar el contenido de Ni (II), en la muestra de cemento estudiada, se han seguido dos procedimientos, según se utilice medio amoniacal o medio cianurado:

\section{a) Medio amoniacal}

Se toman $25 \mathrm{~m} l$ de la disolución preparada según 3.4. (indistintamente clorhídrica o sulfúrica) y se añade hidróxido de amonio hasta conseguir un $\mathrm{pH}$ comprendido entre 9 y 10 . Se filtra y se afora la disolución a $50 \mathrm{~m} l$ con agua permutada y bidestilada.

\section{b) Medio cianurado}

Se toman $25 \mathrm{ml}$ de la disolución preparada según 3.4. (indistintamente clorhídrica o sulfúrica), se añade hidróxido de sodio hasta conseguir un $\mathrm{pH}$ de 12 a 13 (aparece un precipitado de color marrón-rojizo, soluble en exceso de cianuro) y, a continuación, cianuro de potasio hasta disolución del precipitado, en cuyo momento la disolución adquiere un color amarillo. Por último, como en el procedimiento anterior, la disolución se afora con agua permutada y bidestilada a $50 \mathrm{ml}$.

\section{c) Obtención de los polarogramas}

Se toma $1 \mathrm{ml}$ de cualquiera de las disoluciones anteriores (a o b) y $25 \mathrm{ml}$ de agua permutada y 
bidestilada, que se vierten en la célula polarográfica, procediéndose seguidamente de forma similar a la descrita para la determinación del contenido de Mo (VI). En la figura 3 se ha representado, a título de ejemplo, una determinación de las efectuadas para este fin; el polarograma A corresponde a la disolución preparada del cemento según a), el polarograma $\mathrm{B}$ y los tres siguientes $(\mathrm{C}, \mathrm{D}$ y $\mathrm{E}$ ) a la disolución anterior con una adición de $50 \mu \mathrm{l}$ de la disolución patrón de $\mathrm{Ni}$ (II) $10^{-3} \mathrm{M}$ (equivalente a $5 \times 10^{-8}$ moles) para el primero, de $100 \mu l$ para el segundo, $150 \mu l$ para el tercero y $200 \mu l$ para el cuarto.

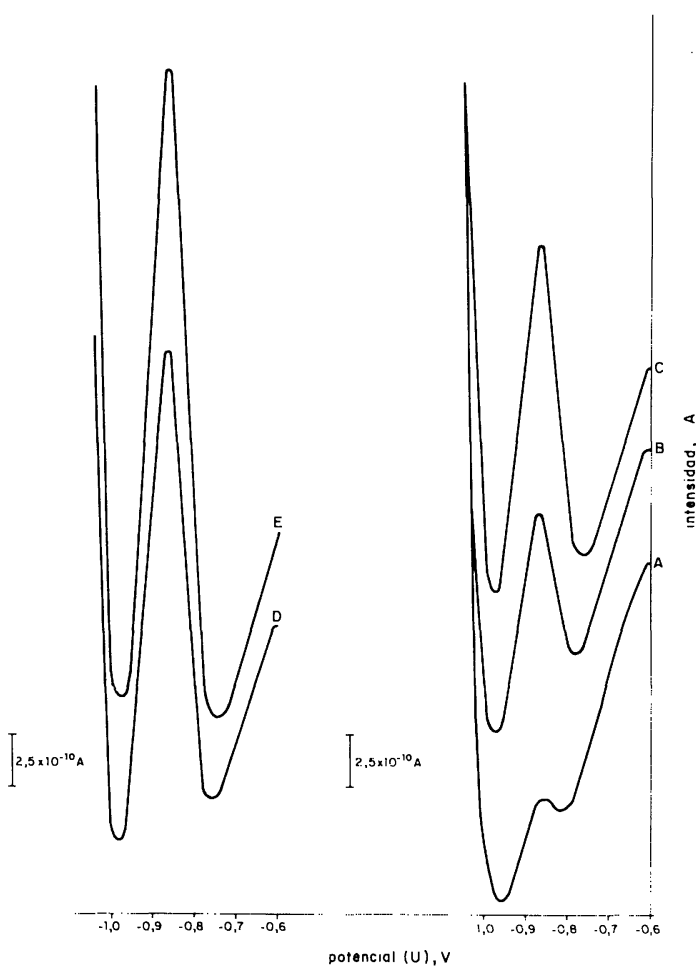

Fig. 3.-Determinación de Ni (II). Polarogramas de: A.-Disolución del cemento, medio amoniacal.

B, C, D y E.-Disolución del cemento, medio amoniacal, más $n 50 \mu l$ de disolución de $\mathrm{Ni}$ (II), $10^{-3} \mathrm{M} . n=1$, para B, a 4, para E.

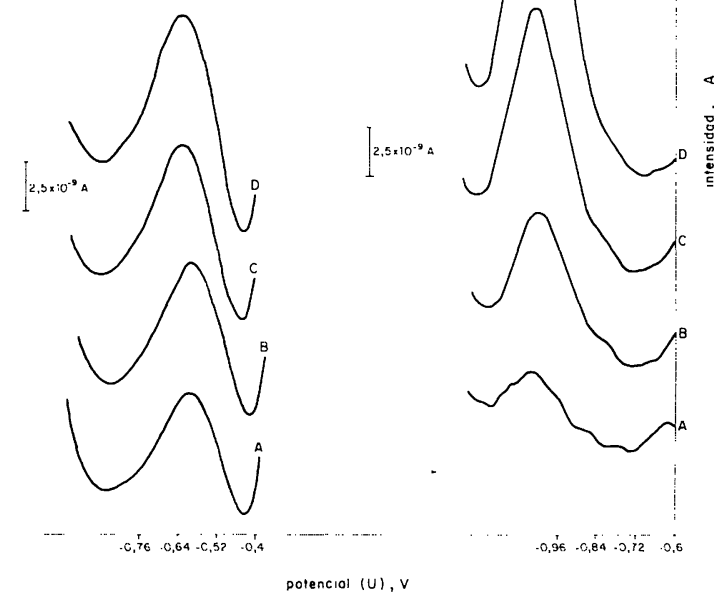

Fig. 4.-Determinación de V (V). Polarogramas de: A.-Disolución del cemento, medio $\mathrm{NaHCO}_{3}$. B, C y D.-Disolución del cemento, medio $\mathrm{NaHCO}_{3}$, más $n 50 \mu l$ de disolución de $\mathrm{V}(\mathrm{V}), 10^{-3} \mathrm{M} . n=1$, para B, a 3, para D

\subsubsection{Determinación del contenido de vanadio (V)}

Se toman $25 \mathrm{~m} l$ de cualquiera de las disoluciones obtenidas en 3.4. Se añade carbonato ácido de sodio hasta conseguir un $\mathrm{pH}=8$; se filtra y con agua permutada y bidestilada se afora a $50 \mathrm{ml}$.

Se llevan a la célula polarográfica $1 \mathrm{~m} l$ de la disolución anterior y $25 \mathrm{~m} l$ de agua permutada y bidestilada, procediéndose, a continuación, como en los casos precedentes para determinar los contenidos de molibdeno (VI) y de níquel (II).

En la figura 4 se han representado, a titulo de ejemplo, dos determinaciones realizadas a diferente impulso y tiempo de gota; el polarograma A corresponde a la disolución preparada del cemento y los polarogramas $\mathrm{B}$ y siguientes $\left(\begin{array}{l}\mathrm{C} \\ \text { y }\end{array}\right.$ $50 \mu \mathrm{l}$ de la disolución patrón de vanadio $(\mathrm{V}) 10^{-3} \mathrm{M}$ (equivalente a $5 \times 10^{-8}$ moles) para el primero, 100 y $150 \mu l$, respectivamente, para los otros dos polarogramas. 


\subsubsection{Resultados y discusión}

Los resultados se han calculado a partir de las alturas de pico obtenidas de los polarogramas correspondientes a las disoluciones originales de la muestra de cemento y de los polarogramas de dichas disoluciones con cada una de las adiciones de las disoluciones patrón, extrapolando a altura de pico igual a cero; finalmente, dichos resultados se refieren a la masa de la muestra.

Los resultados obtenidos para el níquel (II) en medio amoniacal y en medio cianurado han sido análogos.

Los resultados obtenidos, en p.p.m., ordenados de mayor a menor son los que se incluyen en la tabla 1 para las $n$ determinaciones realizadas de cada uno de los iones mencionados, así como el valor medio $(\bar{X})$ y la desviación típica $(\sigma)$.

\section{CONCLUSIONES}

\section{Primera}

Los resultados obtenidos, en el presente trabajo, han confirmado y puesto de manifiesto que la polarografia diferencial de impulsos se puede aplicar para determinar cuali y cuantitativamente los compuestos a base de molibdeno (VI), níquel (II) y vanadio (V) en los cementos.

\section{Segunda}

Por medio de la polarografía diferencial de impulsos se han podido determinar, en la muestra de cemento estudiada, los contenidos de $\mathrm{Ni}$ (II), de V (V) y de Mo (VI), que se encuentran comprendidos entre 2,9 y 3,4 p.p.m. $\left(0,0002_{9}\right.$ y $0,0003_{4} \%$, en masa), 20,2 y 30,6 p.p.m. $(0,0020$ y $0,0030 \%$, en masa) y 91 y 120 p.p.m. (0.0091 y $0,0120 \%$, en masa), respectivamente.

\section{Tercera}

A la vista de los resultados obtenidos en este trabajo se piensa continuar con el estudio iniciado, con el fin de poder perfeccionar los procedimientos operatorios, de un modo especial, para determinar los contenidos de Mo (VI) y V (V) en los cementos.

\section{BIBLIOGRAFIA}

(1) A. COBO y P. SANCHEZ BATANERO: Afinidad XXXVIII (374), (1981).

(2) DEANE, P. G.: Zement-Kalk-Gips, 10, 526-529, (1982).

(3) M. von STACKELBERG, P. KLINGER, W. KOCH, and E. KRATH: Tech. Mitt. Krupp Forschungsber. 2, 59, (1939).

(4) F. A. UHL: Zeit. and Chem. 110, 102 (1937).

(5) M. I. NICHOLS and L. H. ROGERS: Ind. Eng. Chem. Anal. Edn., 16, 137-40, (1944). 
(6) M. G. JOHNSON and R. J. ROBINSON: Anal. Chem. 24, 366-9, (1952).

(7) E. A. KANEVSKII and L. A. SHVARTSBURD: Zavod. Lab. 9, 283, (1940) (CA 34, 5782).

(8) G. P. PROTSENKO and P. N. KOVALENKO: Technol. Prokritii Metal i Methody Kontrolye Proizv. Sb. 122-7 (1962) (CA 59 - 4537 b).

(9) F. PARTANI and M. MIGLORINI: Ric. Sci. Rend. Sez. A 3 (8) 1085-94, (1963) (CA 61-3670 C).

(10) I. M. KOLTHOFF and I. HODARA: J. Electroanal. Chem. 4 (6) 369-381, (1962).

(11) I. M. KOLTHOFF and I. HODARA: J. Electroanal. Chem. 5 (3), 165-170, (1962).

(12) M. KAWAHARA, H. MOCHIZUKI and R. KOJIYAMA: Japan Analyst 8 (1), 25-30, (1959) (AA 7-145).

(13) R. HÖLTJE und R. GEYER: Z. Anorg. Allgem. Chem. 246, 258-74, (1941).

(14) G. S. DESMUKH und J. P. SRIVASTAVA: Z. Anal. Chem. 176, 28-34, (1960).

(15) V. T. ATHAWALE, R. KAlyANARAMAN and K. A. KHASGIWALE: Anal. Chim. Acta 29, 280-2, (1963).

(16) B. K. AFGHAN and R. M. DAGNALL: Talanta 14, 239-243, (1967).

(17) N. A. POlotebNONA and L. A. FURTUNE: Zh. Neorg. Khim. 14 (8), 2143-6, (1969) (CA $71-97737$ X).

(18) R. V. BIKBULATOVA and S. I. SYNYAKOVA: Izv. Akad. Nauk. (2), 48-59, (1969) (CA 73-51608 n).

(19) K. OGURA and Y. ENAKA: Electrochim. Acta 22 (8), 853-7, (1977).

(20) N. S. SHARIPOVA, O. A. SONGINA and R. K. SHARIPOV: Zh. Anal. Khim. 32 (10) 1918-21, (1977) (CA $88-43136 \mathrm{~h}$ ).

(21) B. STACH und K. SCHOENE: Mikrochim. Acta 2 (5-6), 565-74, (1977).

(22) K. OGURA, Y. ENAKA and K. MORIMOTO: Electrochim. Acta 23 (2) 289-92, (1978). .

(23) S. V. LUGOROI and L. D. PAKLEROVA: Zh. Neorg. Khim. 22 (6), 1550-3, (1977) (CA 87-75660 v).

(24) G. PABBU VIJAY, R. ZARAPLEX LAZMAN und M. SANKER: Mikrochim. Acta 2, (1-2), 67-76, (1980).

(25) S. H. EFRAIM, I. MOUSTAFA and I. M. ISMAIL: Anal. Chim. (Rum). 69 (5-6) 219-229, (1979) (AA 39-6 B 9).

(26) T. E. EDMONS: Anal. Chim. Acta 118 (2), 329-333 (1980).

(27) P. LANZA, D. FERRI and P. L. BALDINI: Analyst 105 (249), 379-385, (1980).

(28) S. ZELTER and COLL.: Czech. Chem. Communs, 4, 319, (1932) (I. M. Kolthoff, J. J. Lingane - Polarography - Interscience Publishers - John Wiley and Sons Inc., New York).

(29) M. von STACKELBERG, P. KLINGER, W. KOCH und E. KRATH: Tech. Mitt. Krupp. Forschungsber. 2, 59, (1939).

(30) G. THANHEISER und J. WILLENS: Arch. Eisenhüttnw. 13, 73, (1939).

(31) M. VORISKOVA and COLL.: Czech. Chem. Communs 11, 588, (1939). (I. M. Kolthoff, J. J. Lingane - Polarography Interscience Publishers - John Wiley and Sons Inc., New York).

(32) J. J. LINGANE: J. Am. Chem. Soc. 67, 182, (1945).

(33) J. J. LINGANE and L. MEITES: J. Am. Chem. Soc. 69, 1021, 1882, (1947); 70, 2525 (1948).

(34) F. LUCENA CONDE, J. HERNANDEZ MENDEZ, A. SANCHEZ MISIEGO y M. DELGADO ZAMARREÑO: Ion 36 (414) 1-5, (1976).

(35) M. F. GRIGOR'EVA, L. A. KASATOCHKinA and I. A. TSERKovNitSKAYA,: Zh. Anal. Khim. 32 (6), 1146-8, (1977) (CA 88-57921 x).

(36) T. NOMURA, and G. NAKAGAWA: Talanta 24 (7), 467-9, (1977).

(37) V. RAO, S. NARAYANAI and S. B. RAO: J. Electroanal. Chem. 108 (3), 373-376, (1980).

(38) M. PAVLICK and COLL.: Czech. Chem. Communs 3, 223, (1931).

(39) J. J. LINGANE and H. KERLINGER: Ind. Eng. Chem. Anal. Ed. 13, 77, (1941).

(40) P. R. STOUT und J. LEVY and COLL.: Czech. Chem. Communs 10, 136, (1938).

(41) D. N. HUME and I. M. KOLTHOFF: J. Am. Soc. 73, 4423, (1950).

(42) F. BURRIEL-MARTI, R. GALLEGO-ANDREU y P. PEREZ-CARRETERO: Actas del Simposium de microanálisis de Montreal (Canadá) 843-851, (1961). 
(43) F. BURRIEL-MARTI y F. SAINZ DEL RंIO: An. Real. Soc. Fis. y Quim. B 47, 808, (1951).

(44) O. E. RUVINSKII, A. K. NEVAROVA and V. I. GRINFELD'D: Zh. Anal. Khim.32 (6), 1099-103, (1978) (CA 88-117937 g).

(45) V. V. ASTAFEVA, G. V. PROKLOROVA and R. M. SAliKHDZ-HANOVA: Zh. Anal. Khim. 31 (2), 260-4, (1976) (CA 85-116091 g).

(46) C. KUHNHARDT and W. ANGERMANN: Chem. Anal. (Warsaw) 22 (1), 37-44, (1977) (CA 88-83060 K).

(47) E. A. MAMBETKAZIEW: Dokl. Acad. Nauk SSSR, 234 (5), 1118-21, (1977) (CA 87-75661 w).

(48) O. E. RUVINSKII, N. M. UPENISKAYA and V. E. GODYATSKII: Electrokhimiya 16 (10), 1579-80, (1975) (CA 91-219339 e).

(49) O. E. RUVINSKII and V. N. SIRKO: Electrokhimiya 14 (7), 1043-4, (1978) (CA 89-137465).

(50) I. V. VOITENKO, G. V. PROKHONOVA and P. K. AGASYAN: Zh. Anal. Khim. 35 (4) 702-704, (1980) (AA 40 - I B 161).

\section{publicación del i.e.t.c.c.}

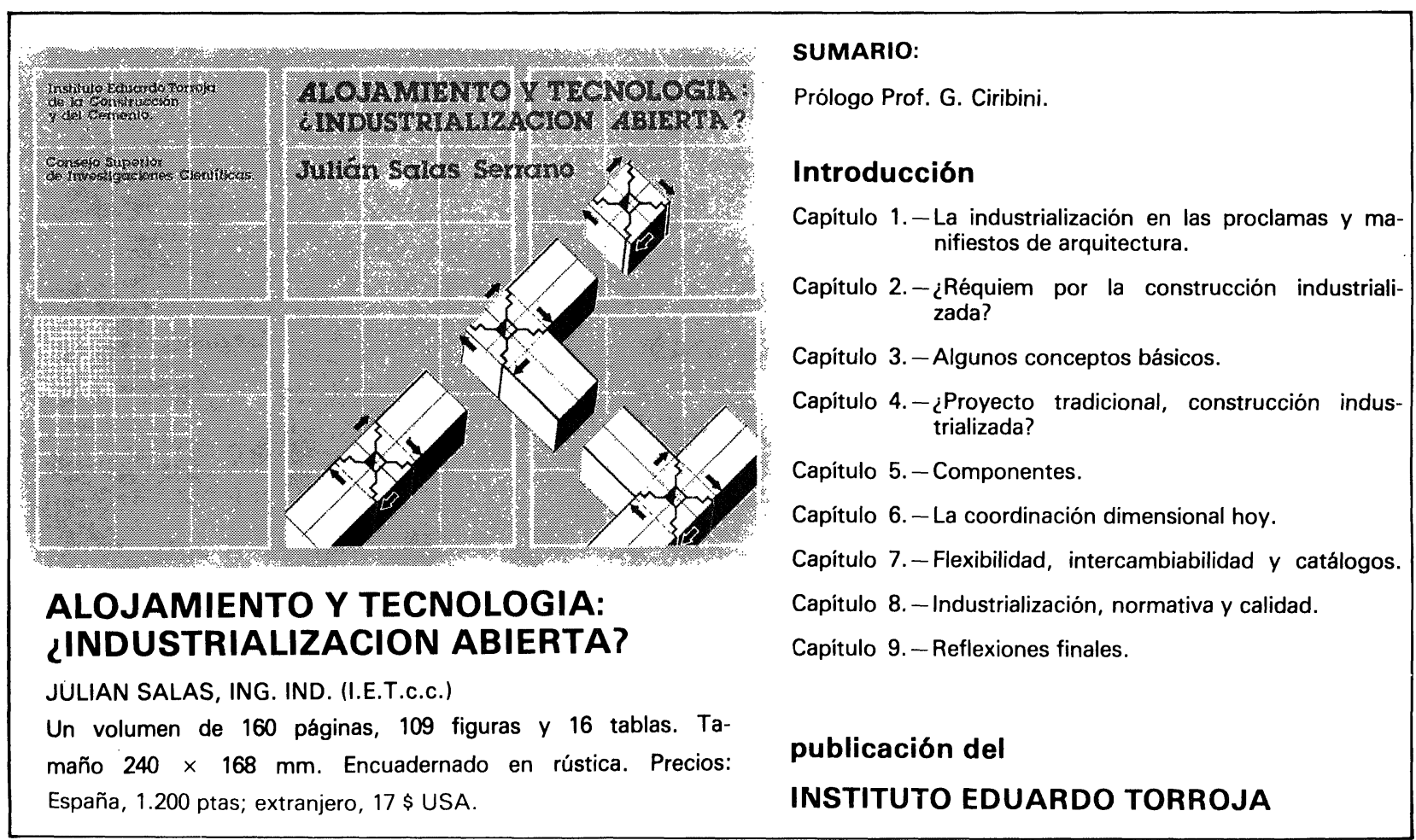

